\title{
Simulación computacional de la transferencia de calor en herramientas usadas en soldadura por fricción-agitación
}

\section{Computer Simulation of Heat Transfer on Tools Used in Friction Stir Welding}

\author{
DAVID STEEVEN VILLA-SALAZAR \\ Estudiante Ingeniería Mecatrónica \\ Instituto Tecnológico Metropolitano \\ david7246@gmail.com \\ Medellín, Colombia \\ DIEGO ANDRÉS HINCAPIÉ-ZULUAGA \\ M.Sc. en Ciencias Física \\ Instituto Tecnológico Metropolitano \\ diegohincapie@itm.edu.co \\ Medellín, Colombia \\ EDWAR ANDRÉS TORRES-LÓPEZ \\ Ph.D Ingeniería Mecánica \\ Universidad de Antioquia \\ eandres.torres@udea.edu.co \\ Medellín, Colombia
}

Fecha de recibido: 20/01/2015

Fecha de aceptado: 09/07/2015

Forma de citar/How to cite: VILLA, D.; HINCAPIÉ, D. y TORRES, E. Simulación computacional de la transferencia de calor en herramientas usadas en soldadura por fricción-agitación. Rev. UIS Ingenierías, 2015, vol. 14, no 2, p.p. 19-26.

DOI: http://dx.doi.org/10.18273/revuin.v14n2-2015003 (c) (1) $\Theta$

\section{RESUMEN}

La Soldadura por Fricción-Agitación es un proceso empleado para la unión de metales, principalmente de baja temperatura de fusión, como aleaciones de aluminio. Los estudios que involucran la herramienta usada en este proceso de soldadura se centran principalmente en cuantificar el efecto que la geometría ejerce sobre las propiedades finales de la junta. En este trabajo se estudia el efecto de la geometría de la herramienta en la disipación de calor, utilizando simulación computacional, específicamente el módulo CFX de Ansys®14.5. Fueron considerados tres geometrías de herramienta, cada una construida de forma modular con tres partes: cono, vástago e implante. El ensamble virtual fue sometido a simulación con modelos para el cálculo de la temperatura y tiempos de calentamiento. Los modelos fueron evaluados considerando como parámetros de soldadura las velocidades de avance de 100, 150, 200 y $250 \mathrm{~mm} \cdot \mathrm{min}^{-1}$ y rotación de 700, 800, 900 y 1000 rpm. Para el cálculo se consideró que la temperatura alcanzada en el implante (hombro y pin) es de $500^{\circ} \mathrm{C}$, con tiempo de soldadura de 1 hora. Los resultados permiten refinar el diseño de herramientas con vástagos más cortos. Se confirma la efectividad del uso de aletas en el vástago para mayor disipación de calor, siendo más efectivo el uso de aletas desplazadas hacia el inserto en comparación con el sistema de aletas con estas en el centro.

PALABRAS CLAVE: Soldadura FSW de aluminio, transferencia de calor, diseño de herramientas, simulación térmica. 


\section{ABSTRACT}

Friction Stir Welding is a process used for joining metals, mainly of low melting temperature, such as aluminum alloys. Studies involving the tool used in this welding process, focusing mainly on quantifying the effect of geometry on the final properties of the welded joint. In this paper it was studied the effect of the tool geometry in the heat dissipation, using computer simulation, specifically the module CFX of Ansys ${ }^{\circledR} 14.5$. They were considered three tool geometries, each built in a modular way with three parts: Collet Chuck, Shank and Implant. The virtual assembly was subjected to simulation models for the calculation of temperature and heating time. The models were evaluated, considering as welding parameters: Welding Speeds of 100, 150, 200 and $250 \mathrm{~mm} \cdot \mathrm{min}^{-1}$ and Rotation Speed of 700, 800, 900 and $1000 \mathrm{rpm}$. For the calculation it was assume that the temperature reached on the implant (Shoulder and Pin) were $500^{\circ} \mathrm{C}$, in a welding time of 1 hour. The results allow to improve the tools design with a shorter shank and allows to confirm the effectiveness of using fins on the shank for greater heat dissipation, being more effective the use of Fins Displaced towards the insert in comparison with System with Fins on the Rod Center.

KEYWORDS: Aluminum Welding, Heat Transfer, Tool Design, Thermal Simulation.

\section{INTRODUCCIÓN}

En 1991 fue desarrollado en el Instituto de Soldadura (The Welding Institute, TWI) un novedoso método denominado soldadura por fricción-agitación (Friction Stir Welding, FSW) [Thomas et al. (1993)]. La FSW se basa en el empleo de una herramienta rotativa, no-consumible, constituida por un hombro y un pin diseñados para el calentamiento y agitación del metal de la junta. Al inicio del proceso la herramienta en rotación es introducida en la junta, produciendo el calentamiento por fricción del metal circundante. El calor generado plastifica, mas no funde, el metal alrededor de la herramienta, la cual posteriormente se desplaza a lo largo de la junta cerrando la brecha entre los materiales [Mishra Ma (2005)].

Las herramientas para la realización de las soldaduras en aleaciones de aluminio han sido estudiadas extensivamente, evaluando el efecto de la geometría de estas sobre la respuesta mecánica y metalúrgica de la junta soldada [Fujii et al. (2006), Mishra Mahoney (2007)]. Entre tanto el diseño de la herramienta no es uno de los puntos más fuertes, a excepción de los trabajos realizados por [Nicholas Thomas (1998) y Fehrenbacher et al. (2014)], para la distribución de temperatura en el pin, y [Xue et al. (2013)] para determinar los esfuerzos generados durante la soldadura. Con relación al efecto de la herramienta sobre el proceso, la literatura presenta un claro vacío [Colligan et al. (2003)].

Es común la realización de FSW empleando una fresadora, la cual permite la realización de esta operación con restricciones como el empleo limitado de velocidades de avance y rotación, además de la falta de control de la penetración de la herramienta [Kuljanic (2005)]. Otro elemento crítico es el sobrecalentamiento que el husillo de la fresadora sufre, pues en el diseño de las herramien- tas no son considerados mecanismos para evitarlo. En este estudio se busca introducir un elemento disipador de calor. La importancia de este elemento radica en la posibilidad de acoplar una fresadora convencional para la realización de uniones soldadas por FSW, además de permitir la disipación del calor generado, evitando un ensamble permanente entre del husillo con la herramienta por causa de la dilatación alcanzada en la herramienta durante la soldadura.

Para estos fines serán diseñadas herramientas destinadas a la soldadura por fricción-agitación de aleaciones de aluminio teniendo en cuenta como parámetro fundamental la disipación de calor de la herramienta. Se evaluaron diferentes geometrías de herramienta, mediante simulación CFX, buscando minimizar el flujo de calor desde la herramienta hacia el husillo de la máquina donde se realizan las soldaduras, con una longitud de vástago mínima para reducir el torque generado en la herramienta debido a la acción de la fuerza axial.

\section{MÉTODOS}

En este trabajo fue analizado el comportamiento de una herramienta modular para la realización de soldaduras por fricción-agitación de aluminio, la cual está constituida por tres elementos: cono morse, vástago e inserto. El cono morse permite el montaje de la herramienta en el husillo de la fresadora, el vástago es el elemento disipador de calor y el inserto es el responsable de llevar a cabo la soldadura por fricción. El diseño y ensamble de la herramienta fue realizado utilizando el software Siemens NX 8.0.0.25. El cono y el inserto, son los mismos en todo el proceso de simulación; mientras el vástago será el elemento que sufrirá cambios geométricos. Un ejemplo de herramienta modular y su ensamble se presenta esquemáticamente en la figura 1a, mientras en la figura $1 \mathrm{~b}$ 
aparecen las superficies de contacto de los diferentes elementos. Para el diseño de los vástagos fueron consideradas tres geometrías: un vástago completamente liso, uno con ranuras en el centro y otro con aletas desplazadas hacia el inserto. La diferenciación entre aletas y ranuras en este trabajo se sostiene con base en el grado de turbulencia en el aire que ambos elementos pueden provocar, así como por el hecho que el volumen de aire dentro de la ranura es menor con relación a las aletas, lo que permitiría, en principio, la mejor disipación de calor por parte de este último. La herramienta con vástago liso será considerada la referencia para verificar el comportamiento de las otras herramientas; los vástagos con ranuras en el centro y con aletas desplazadas deben disipar más calor que la herramienta con vástago liso, lo cual permite verificar la coherencia de los resultados de la simulación. El efecto de las aletas desplazadas en el comportamiento de disipación de calor en el vástago es indeterminado.

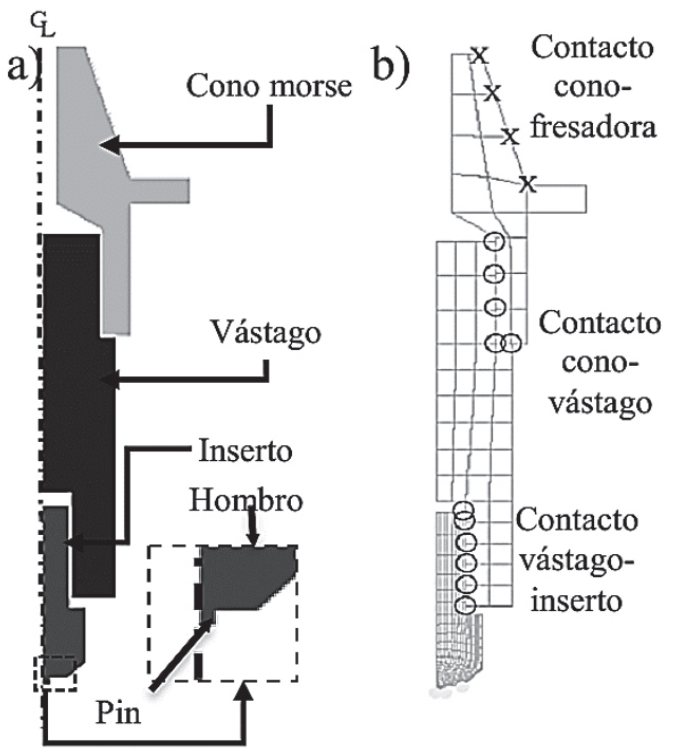

Figura 1. Herramienta modular. (a) Partes de la herramienta: cono, vástago e inserto. (b) Contactos entre los diferentes elementos: Herramienta-herramienta (O) y herramienta-fresadora (X). Fuente: Adaptado de [Dickerson et al. (2003)].

Las dimensiones de las herramientas diseñadas se muestran en la figura 2. Para las herramientas se determinaron límites dimensionales, donde el diámetro mínimo fuese de $24 \mathrm{~mm}$ mientras el máximo no sobrepasara los 38 $\mathrm{mm}$. Estos límites corresponden al diámetro del inserto $(24 \mathrm{~mm})$ y el de la barra de metal a partir del cual será fabricado el vástago (1,5 pulgadas). La herramienta lisa fue generada con el di'ametro máximo en todo su vástago, la herramienta con ranuras tiene como diámetro máximo $38 \mathrm{~mm}$, con diámetro interno de las ranuras de $24 \mathrm{~mm}$, mientras que la herramienta con aletas desplazadas fue construida con el diámetro mínimo de $24 \mathrm{~mm}$ con sus protrusiones de diámetro $38 \mathrm{~mm}$. La longitud de los vástagos se asumió de $240 \mathrm{~mm}$, lo cual resultó suficiente para verificar la disipación del calor de forma tal que el extremo en contacto con el cono morse alcanzara una temperatura de $50^{\circ} \mathrm{C}$, la cual es una temperatura aceptable para evitar el calentamiento del husillo de la fresadora.

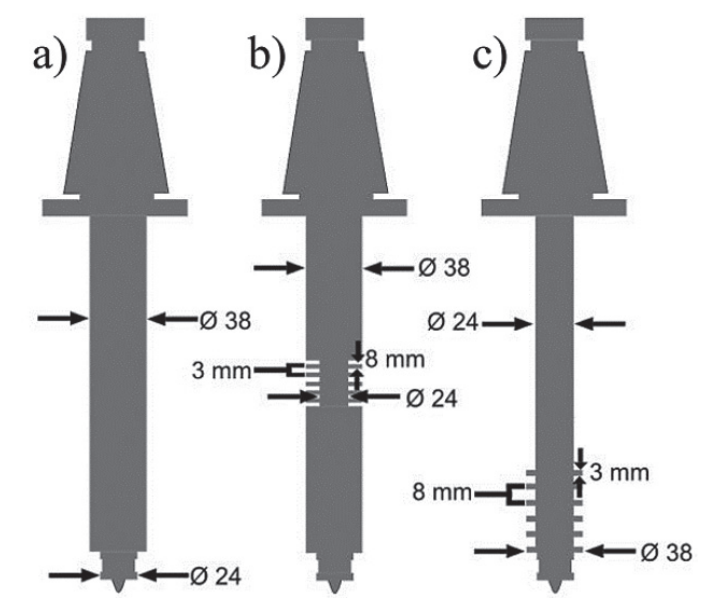

Figura 2. Dimensiones de las geometrías generadas. (a) Herramienta lisa. (b) Herramienta con ranuras. (c) Herramienta con aletas.

Para la simulación de las herramientas el sistema fue dividido en cuatro partes: las tres secciones de la herramienta (figura 2) y un cubo de aire que abarca todo el vástago, con el fin de recrear el entorno en que se desarrollara la soldadura (ver figura 3a), el cual corresponde a aire en condiciones atmosféricas. El cubo se definió como fluido ya que representa el elemento aire como gas ideal en el módulo CFX. En esta simulación se considera el movimiento rotacional de la herramienta, pero donde el desplazamiento de la misma es realizado por la junta a soldar y el aire entorno; por lo tanto, la velocidad de avance de la soldadura corresponde a la velocidad de ingreso del aire al cubo. En el cubo fueron definidos cuatro límites principales: una cara lateral corresponde a un límite tipo Inlet, que reproduce la entrada de aire al sistema. Las tres caras laterales restantes se usan como límites tipo Opening, que permiten la circulación libre del aire.

En la parte inferior, la cual es la superficie que se encuentra por fuera del cubo, se definió un límite tipo Wall que reproduce el calor resultante de la fricción entre el hombro-pin con la junta. Para determinar la condición de esta frontera fueron consideradas medidas de temperatura durante el proceso de soldadura para aleaciones 
de aluminio, las que indican que el pico máximo en la región soldada (entercara herramienta-junta) oscila entre $\operatorname{los} 450^{\circ} \mathrm{Cy}$ los $550^{\circ} \mathrm{C}$ [Hwang et al. (2008), Xu et al. (2009)].

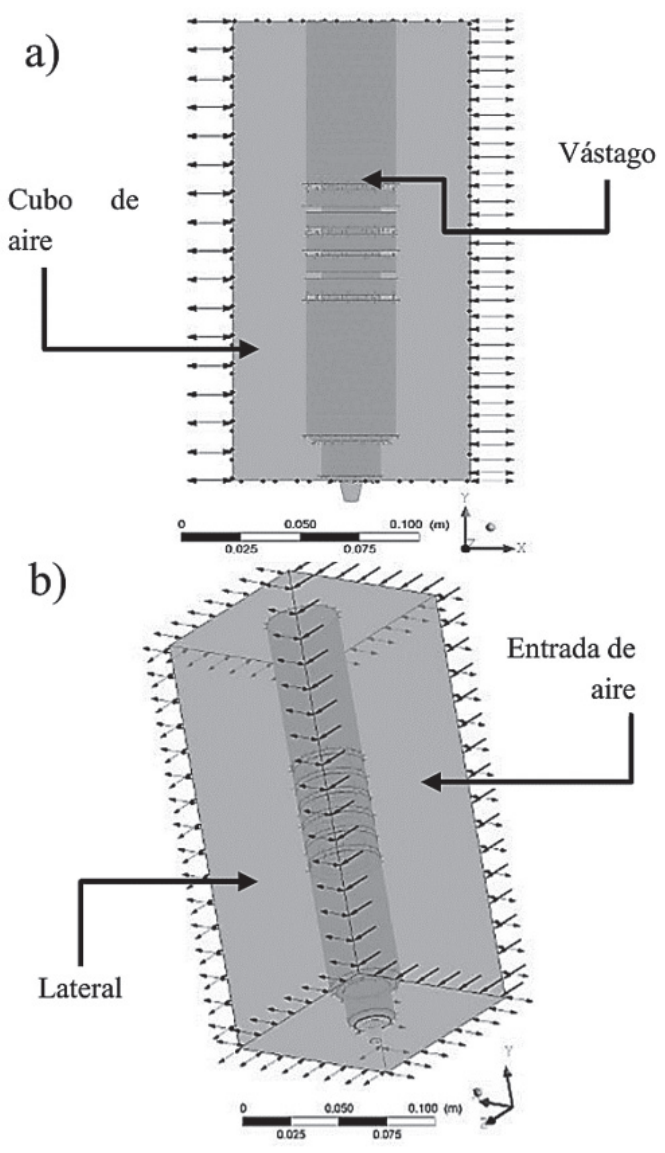

Figura 3. (a) Dominios usados para la simulación: cubo y vástago. (b) Inter-caras del cubo de aire, y límite tipo Wall creado para el ingreso de calor en el sistema.

A pesar de que la temperatura varía en función de los parámetros de soldadura, se consideró que una buena aproximación para la temperatura en el hombro y el pin corresponde a $500^{\circ} \mathrm{C}$, valor que será empleado en la simulación del calentamiento de la herramienta. Los detalles de los dominios para la simulación se aprecian en la figura 3 b. Las mallas usadas en cada herramienta tienen una variación mínima entre ellas. La tabla1 muestra los datos de las métricas de cada geometría, siendo empleados tetraedros aplicados mediante un método independiente de la geometría.

La simulación del proceso se realiza considerando estado transiente, definiendo un tiempo estándar de una hora, con cálculos cada minuto, lo que implica calcular 60 timesteps. Cada uno de los elementos de la herramienta: cono, vástago e inserto fueron fabricados con materiales diferentes. Las características de cada uno de estos materiales son presentadas en la tabla 2 . Para la simulación se consideró que las propiedades son constantes, ya que las temperaturas alcanzadas son inferiores a $500^{\circ} \mathrm{C}$.

Tabla 1. Métricas de las mallas para la simulación térmica de las herramientas.

\begin{tabular}{|c|c|c|}
\hline \multirow{2}{*}{ Geometría } & Min. & Max. \\
\cline { 2 - 3 } & \multicolumn{2}{|c|}{ Oblicuidad } \\
\hline Lisa & $8,82 \mathrm{E}-04$ & 0,6880 \\
\hline Ranuras & $4,81 \mathrm{E}-04$ & 0,6954 \\
\hline Aletas & $1,54 \mathrm{E}-03$ & 0,6708 \\
\hline & \multicolumn{2}{|c|}{ Rel. Aspecto } \\
\hline Lisa & 1,1767 & 5,2275 \\
\hline Ranuras & 1,1716 & 5,9749 \\
\hline Aletas & 1,1718 & 5,4370 \\
\hline & \multicolumn{2}{|c|}{ Calidad Ortogonal } \\
\hline Lisa & 0,4377379 & 0,9906732 \\
\hline Ranuras & 0,3864439 & 0,9915113 \\
\hline Aletas & 0,4269176 & 0,9876827 \\
\hline & Elementos & Nodos \\
\hline Lisa & 3.215 .913 & 555.820 \\
\hline Ranuras & 1.943 .238 & 350.883 \\
\hline Aletas & 2.156 .409 & 379.733 \\
\hline
\end{tabular}

Tabla 2. Características de los materiales usados para la construcción de las herramientas de FWS. C.T. (Conductividad Térmica). C.E (Calor Específico). E.T. (Expasión Térmica).

\begin{tabular}{|c|c|c|c|}
\hline Elemento & Cono & Vástago & Inserto \\
\hline \multirow{2}{*}{ Material } & $\begin{array}{c}\text { AISI-SAE } \\
4340\end{array}$ & $\begin{array}{c}\text { AISI-SAE } \\
1045\end{array}$ & $\begin{array}{c}\text { AISI-SAE } \\
\mathrm{H} 13\end{array}$ \\
\hline \multirow{2}{*}{ C.T. } & 44,5 & 49,8 & 24,4 \\
& $\mathrm{~W} / \mathrm{m}-\mathrm{K}$ & $\mathrm{W} / \mathrm{m}-\mathrm{K}$ & $\mathrm{W} / \mathrm{m}-\mathrm{K}$ \\
\hline \multirow{2}{*}{ C.E. } & 0,475 & 0,489 & 0,460 \\
& $\mathrm{~J} / \mathrm{g}-{ }^{\circ} \mathrm{C}$ & $\mathrm{J} / \mathrm{g}-{ }^{\circ} \mathrm{C}$ & $\mathrm{J} / \mathrm{g}-{ }^{\circ} \mathrm{C}$ \\
\hline \multirow{2}{*}{ E.T. } & 14,5 & 14,0 & 12,4 \\
& $\mu \mathrm{m} / \mathrm{m} .{ }^{\circ} \mathrm{C}$ & $\mu \mathrm{m} / \mathrm{m} .{ }^{\circ} \mathrm{C}$ & $\mu \mathrm{m} / \mathrm{m} .{ }^{\circ} \mathrm{C}$ \\
\hline
\end{tabular}

Finalmente, las variables parametrizadas son la velocidad de rotación de la herramienta y la velocidad de avance (o de entrada del aire al cubo) definida en el límite tipo Inlet del dominio Aire. Se usaron cuatro velocidades de avance y cuatro velocidades de rotación las cuales se indican en la tabla 3 , siendo simuladas 16 combinaciones. 
Tabla 3. Parámetros de soldadura considerados para la simulación.

\begin{tabular}{|c|c|c|c|c|}
\hline Velocidad & $\mathbf{1}$ & $\mathbf{2}$ & $\mathbf{3}$ & $\mathbf{4}$ \\
\hline Rotación $(\mathrm{rpm})$ & 700 & 800 & 900 & 1000 \\
\hline Avance $\left(\mathrm{mm} \cdot \mathrm{min}^{-1}\right)$ & 100 & 150 & 200 & 250 \\
\hline
\end{tabular}

Para la adquisición de datos se definieron cinco regiones, delimitadas por seis planos como se muestra en la figura4.

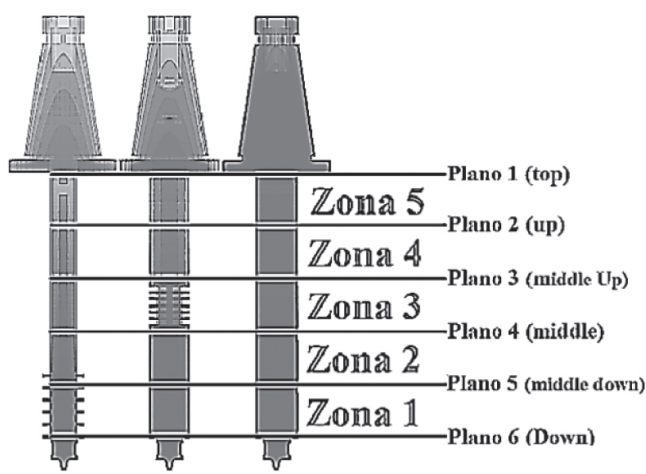

Figura 4. Distribución de zonas y planos en donde se adquirieron los datos. La diferencia en tonos de gris está relacionado con la temperatura alcanzada, donde el gris más intenso corresponde a $500^{\circ} \mathbf{C}$.

Para adquirir los datos transientes en función del tiempo en cada uno de los seis planos se crearon expresiones para el cálculo de promedios de área respecto a la temperatura, luego estas expresiones fueron llevadas a un gráfico de temperatura contra tiempo, para comparar el comportamiento entre zonas y exportar los datos como tablas CSV (commaseparatedvalues) y convertidas a formato .xls de Microsoft Excel@).

Para la comparación y traficación de los datos obtenidos, se escribió un código en Matlab®, que hace un análisis entre los valores de temperatura en el último instante de tiempo de cada combinación de parámetros. Este proceso se realiza en cada plano para facilitar la comparación entre herramientas, pero además permite graficar el cambio de temperatura en cada zona, para evaluar el efecto que cada elemento disipador de calor ejerce en el vástago. El código permite comparar los cerca de 17.280 datos obtenidos en la simulación.

\section{RESULTADOS Y ANÁLISIS}

Para comenzar, se presenta en la figura 5 la distribución de temperatura determinada por medio de simulación com- putacional CFD, considerando un modelo transciente, para el instante $t=1$ hora. Los resultados confirman algunos hechos esperados, respecto al efecto de la geometría en la distribución de la temperatura, como la mayor disipación de calor por las aletas y menor para el caso del vástago liso.

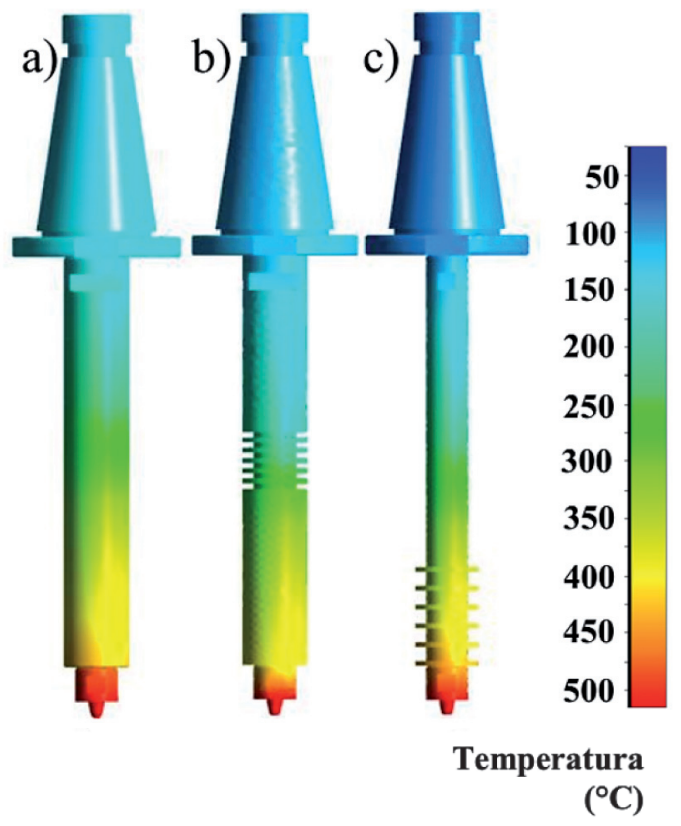

Figura 5. Distribución de la temperatura en las herramientas para FSW diseñadas para la unión de aleaciones de aluminio, considerando la temperatura para el instante $t=1$ hora. (a) Herramienta lisa. (b) Herramienta con ranuras. (c) Herramienta con aletas. La temperatura en cada punto es determinada por medio de la barra de colores.

Es importante resaltar que se determinó la temperatura en la intercara entre el vástago y el cono morse; los valores determinados, corresponden a 142,110 y $91^{\circ} \mathrm{C}$ para las herramientas lisa, ranura y aletas, respectivamente. Esto indica que en principio la herramienta más adecuada para el desarrollo de soldaduras por FSW sería la herramienta con aletas. También es importante resaltar como para el vástago liso el calor se concentra en el inserto, lo cual puede ser muy interesante pues reduce la pérdida de calor de la junta a través de la herramienta, debido a la menor diferencia de temperatura entre ambos elementos.

Igualmente la diferencia de temperatura $(\Delta T)$ más significativa en todo el conjunto se presenta en la intercara vástago-inserto, con una disminución gradual de la temperatura en el vástago. En el caso de la herramienta con ranuras se observa un mayor gradiente de temperatura en el inserto, lo que significa una mayor pérdida de calor desde la junta, lo que se puede traducir en la necesidad 
de aumentar la velocidad de rotación o disminuir la velocidad de avance, para mantener la temperatura en la junta durante la aplicación de la soldadura.

En el caso de la herramienta con aletas el salto de temperatura entre el inserto y el vástago es menos drástico, pero se observa un efecto significativo en las ranuras, las cuales actúan como disipadores del calor. Finalmente para el caso de la herramientas con aletas desplazadas hacia el inserto, el efecto disipador de las aletas no está en discusión, pero si la posición de estas en el vástago. Para el caso de las aletas cerca a la fuente de calor, éstas permiten concentrar un poco más de temperatura en el inserto, con relación al caso anterior, lo que reduce la fuga de calor por la herramienta. En este caso el $\Delta T$ entre inserto y vástago es menor que en el primer caso, y la distribución de temperatura en el vástago también es más gradual con relación al vástago con ranuras.

El caso anterior representa un instante $t$ en el proceso. Sin embargo, para evaluar el comportamiento de las herramientas durante la ejecución de las soldaduras durante una hora, es necesario establecer la temperatura en instantes de tiempo diferentes. Al determinar las curvas de cambio de temperatura contra tiempo, el $\Delta T$ entre cada región varía. Para verificar dicho efecto, fueron comparados dos instantes de tiempo a $500 s$ y $3000 s$, las cuales corresponden a las líneas verticales punteadas en las figuras 6 y 7. Para la herramienta lisa (figura 6a), a 500 $s, \Delta T$ disminuye significativamente, a medida que transcurre el tiempo, lo que representa una estabilización del paso en que aumenta la temperatura en esta. La caída más significativa del delta de temperatura se presenta en la zona 1 . Al pasar de $500 s$ a $3000 s, \Delta T$ se hace cada vez menor, siendo que, a partir de la zona 2 , la diferencia de cambio es similar entre zonas. Con la herramienta de ranuras (figura 7a), en la zona 3 se observa un aumento súbito en $\Delta T$, lo que indica que las ranuras actúan como acumulador de calor.

Se produce un efecto de resistencia térmica, debido a la disminución de la secciónn transversal del eje del vástago, por la presencia de las ranuras; adicionalmente, al disminuir la diferencia de temperatura, el flujo de calor también disminuye. Esto muestra un claro impacto en el plano Top que representa el sitio donde se encuentra la junta entre el vástago y el cono morse este comportamiento se visualiza en la figura $7 b$; la pendiente de cambio en este plano es notablemente menor que la mostrada en la figura $6 b$.

Finalmente, para la herramienta con aletas (figura 8a) el comportamiento es similar al observado en la herramienta lisa (figura 6a). Las aletas no acumulan calor como las

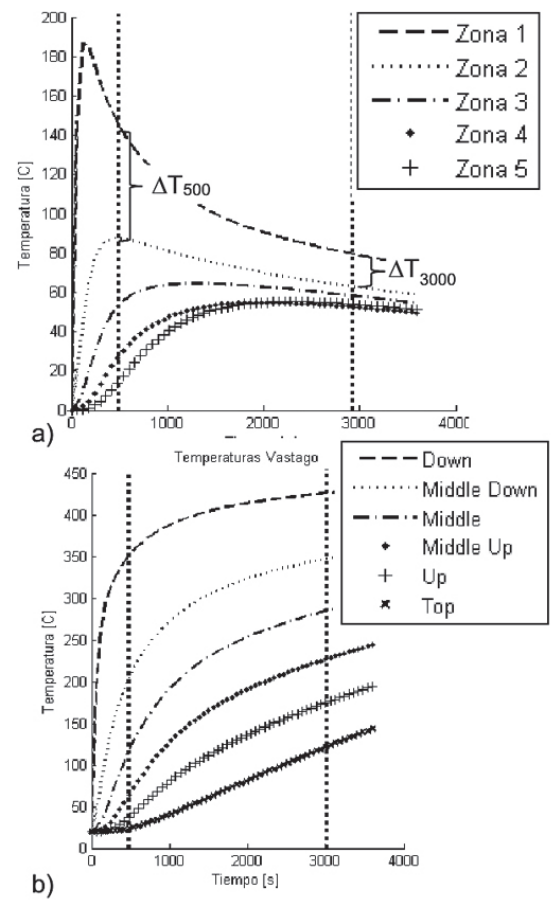

Figura 6. Herramienta lisa: (a) Tasa de aumento de temperatura. (b) Temperatura alcanzada.
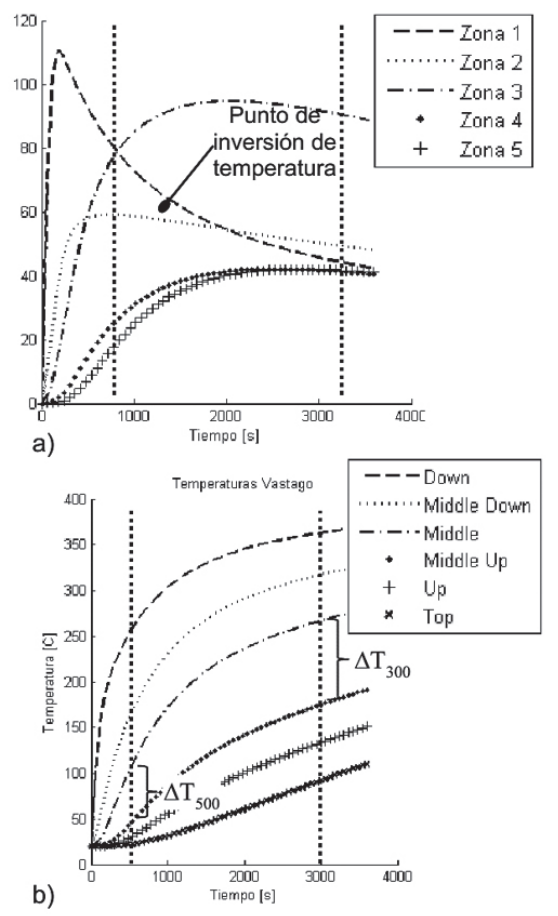

Figura 7. Herramienta con ranuras: (a) Tasa de aumento de temperatura. (b) Temperatura alcanzada. 
ranuras, pero sí atenúan la cantidad la cantidad de calor desprendida hacia el aire; esto se puede observar al comprar los picos de temperatura de la zona 1 de figura 6 a $y$ la figura 8a. A pesar de la temperatura máxima ser idéntica, la herramienta con aletas requiere de más tiempo para alcanzar dicho pico, lo que indica que esta consigue disipar más calor. El factor clave es comparar la figura $6 \mathrm{~b}$ y la figura $8 \mathrm{~b}$ prestando especial atención al plano Top. Es evidente que el objetivo planteado de disipar una mayor cantidad de calor se ve alcanzado con esta geometría, al denotar una pendiente de acumulación de calor bastante más baja, este resultado se da principalmente porque en el resto de la herramienta se ve concentrado mayor parte del calor, dado que las aletas retienen una gran cantidad de este y se va liberando a una tasa más baja, teniendo como escape el aire circundante y el volumen de la herramienta, siendo este último menos afectado que en las dos anteriores geometrías.

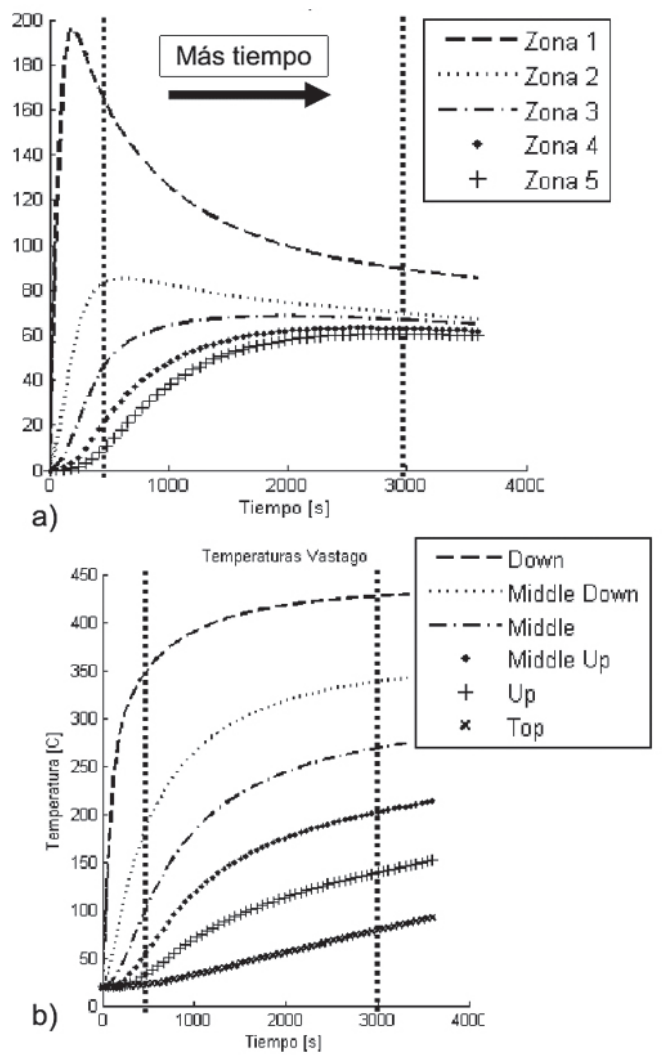

Figura 8. Herramienta con aletas: (a) Tasa de aumento de temperatura. (b) Temperatura alcanzada.

Los resultados confirman la afirmación hecha por [Dickerson et al. (2003)] el cual afirma que las ranuras estabilizan el sistema de forma eficaz; sin embargo, en ese trabajo no se estableció como funcionaban las ranuras.

Los resultados aquí presentados indican que las ranuras actúan como resistencia térmica reduciendo el flujo de calor de la fuente hacia la fresadora, teniendo una gran relevancia el efecto dado a causa del punto de inversión mostrado en la figura 7a que como reacción refleja una diferencia de temperatura acumulada entre los planos Middle y Middle Up. Por otra parte, se confirmó que la herramienta con aletas desplazadas hacia el hombro se comporta de forma idéntica a la herramienta lisa, salvo que las aletas presentan un mejor efecto disipador del calor.

Cabe resaltar que los resultados y el análisis realizados en este trabajo, no de penden de las características de la máquina herramienta. Sin embargo se debe considerar que el diseño del cono morse si depende de la configuración geométrica del equipo (fresadora) que se fuese a utilizar, donde sería necesaria la construcción de este aditamento para la implementación de este proceso en otra fresadora.

\section{CONCLUSIONES}

Fueron diseñadas y evaluadas tres geometrías de herramientas para la realización de soldaduras por medio del proceso de fricción-agitación (FSW), para juntas de aluminio. Fueron seleccionadas tres herramientas: lisa, con ranuras y con aletas, en las cuales se aplicaron con éxito modelos virtuales para evaluar el efecto de la geometría en la disipación del calor generado durante el proceso de soldadura.

Para la herramienta con ranuras fue observado un retraso en el aumento de la temperatura, en la región correspondiente a las ranuras; este fenómeno es asociado a la resistencia térmica ejercida por los surcos y por la disminución de la diferencia de temperatura en la región, siendo esta diferencia la fuerza impulsora para el flujo de calor.

Con la implementación de aletas en la herramienta se produce de forma más eficiente la disipación de calor. La diferencia del efecto entre ranuras o aletas, radica en que el primero aumenta la resistencia al flujo de calor a travás del vástago, mientras el segundo aumenta la cantidad de calor transferido al ambiente, extendiendo el tiempo necesario para el calentamiento de la misma. 


\section{REFERENCIAS}

Colligan, K. J.; Konkol, P. J.; Fisher, J. J.; Pickens, J. R. Friction stir welding demonstrated for combat vehicle construction. Welding Journal, 82, 34-40, 2003.

Dickerson, T.; Shercliff, H. R.; Schmidt, H. Heat flow into friction stir welding tools. En 4th international symposium on friction stir welding, Park City, Utah, USA (p. 16). volumen 14, 2003.

Fehrenbacher, A.; Schmale, J. R.; Zinn, M. R.; Pfefferkorn, F. E. Measurement of tool-workpiece interface temperature distribution in friction stir welding. Journal of Manufacturing Science and Engineering, 136, 021009, 2014.

Fujii, H.; Cui, L.; Maeda, M.; Nogi, K. Effect of tool shape on mechanical properties and microstructure of friction stir welded aluminum alloys. Materials Science and Engineering: A, 419, 25-31, 2006.

Hwang, Y.-M.; Kang, Z.-W.; Chiou, Y.-C.; Hsu, H.-H. Experimental study on temperature distributions within the workpiece during friction stir welding of aluminum alloys. International journal of machine tools $\mathcal{E}$ manufacture, 48, 778-787, 2008.

Kuljanic, E. Amst'05 advanced manufacturing systems and technology: Proceedings of the seventh international conference. chapter Low-Cost Transmation of a Conventional Milling Machine Into a Simple FWS Work Station. Springer Vienna, 2005.

Mishra, R.; Ma, Z. Friction stir welding and processing. Materials Science and Engineering: R: Reports, 50, 1-78, 2005.

Mishra, R.; Mahoney, M. Friction stir welding and processing. chapter 6, 2007.

Nicholas, E.; Thomas, W. A review of friction processes for aerospace applications. International Journal of Materials and Product Techno$\log y$, 13, 45-55, 1998.

Thomas, W.; Nicholas, E.; Needham, J.; Murch, M.; Temple-Smith, P.; Dawes, C. Improvements relating to friction welding, 1993. WO Patent App. PCT/GB1992/002,203.

Xu, W.; Liu, J.; Luan, G.; Dong, C. Temperature evolution, microstructure and mechanical properties of friction stir welded thick 2219-o aluminum alloy joints. Materials E Design, 30, 1886-1893, 2009.

Xue, C.; Ren, X.; Zhang, Q. Heat production simulation and heat-force couple analysis of fsw pin. Engineering Review, 33, 85-91, 2013. 